\title{
Cryptocurrency as a New Financial and Legal Instrument: Defining Cryptoassets in Property Law
}

\author{
Irina Astrakhantseva ${ }^{1, *}$, and Roman Astrakhantsev ${ }^{2}$ \\ ${ }^{1}$ Ivanovo state University of chemistry and technology, 153000 Ivanovo, Russia \\ ${ }^{2}$ National Research University Higher School of Economics, 101000 Moscow, Russia
}

\begin{abstract}
The scientific research study is about the approach to crypto assets in property law. The cryptocurrency, possessing the signs of many civil rights, at the same time does not belong to any of them and does not correspond with anyone in a complete way. This is due to the uniqueness of cryptocurrency as a phenomenon itself. Cryptocurrencies can also represent different types between cryptocurrency holders, as well as between holders and creators of the platform, which have been concluded by implicit actions. The authors propose new definition for cryptocurrency through the cryptographically protected property concept, which has the feature of cryptographic authentication, decentralization, management through consensus, the use of distributed ledgers. According to the authors, the starting point for determining the ownership of the cryptocurrency should be that the subject of the right will be the asset's owner if he or she legally gained access to the private key, by analogy as the subject acquired the ownership of a tangible asset on a legal basis.
\end{abstract}

\section{Introduction}

In 2008, a user calling himself Satoshi Nakamoto published a file "Bitcoin: A Peer-toPeer Electronic Cash System" with the description of the protocol and the payment system operation principle in the form of a decentralized network, further known in the world as Bitcoin. This system used Blockchain technology to conduct the transactions between the users. In fact, bitcoin is the first practical implementation of blockchain technology. It is thanks to this payment system that the blockchain has become widespread and has shown the possibility of creating a distributed payment system without a control center, based only on cryptographic protocols.

According to some studies, by 2021, investment in blockchain research could reach $\$ 9.2$ billion. There is a reason to believe that this technology will bring decentralized trust, transparency and efficiency to the society, despite the existing challenges in the form of scaling and privacy.

\footnotetext{
*Corresponding author: i.astrakhantseva@mail.ru
} 
For the purposes of this study, a blockchain is a peer-to-peer cryptographically protected distributed, (virtually) rewritable registry that supports only the addition of blocks, updated only as a result of an agreement between all the participants [1]. Thus, the blockchain as a registry will have the following properties such as decentralization, distribution, security, adding blocks in chronological order, consensus. From the point of view of the business system, the blockchain is a digital platform, the participants of which can exchange the assets, tokens, electronic money, claims and other objects through the transactions without the participation of a centralized body.

Blockchain technology provides the creation and circulation of digital assets, an important feature of which is their existence exclusively in electronic form in isolation from the material world in the form of a cryptographic digital code. This is precisely their legal uncertainty in the interpretation as an object of law, including in terms of protection [2].

Blockchain systems and distributed registry technologies are new accounting tools that provide distributed accounting without a single controlling party. Such infrastructures make it possible to create: initially digital assets, i.e. the assets that exist only in digital form in the information system issuing them; tokenize existing assets, that is, digitally represent the assets, including rights outside the information system.

Currently, there are significant gaps in the world and Russian legislation governing the circulation of cryptocurrencies. The use of blockchain, smart contracts, tokens and cryptocurrencies is still in a zone of economic uncertainty. Finding cryptocurrency in the gray zone is a deterrent to the introduction of innovative technology. One of the obstacles to legal regulation and uniform policy formation for the emerging crypto asset and blockchain industry is the lack of clear and common terminology. Even the concept of cryptocurrencies does not have a single definition and is interpreted in most sources as a general term for digital tokens that are issued and circulated in distributed ledger systems.

\section{Materials and methods}

The first bitcoin block was created on January 1, 2009 [3]. Bitcoin protocols were in the public domain with an open license, so other options for digital currencies (altcoins) began to appear on its basis. Each of the altcoins has its own blockchain. The most popular and second largest by capitalization (more than $\$ 26$ million as of the date of this study [4]) cryptocurrency is Ethereum. The key difference of this cryptocurrency is the development of a Turing-complete language that allows you to create the software of any complexity, including smart contracts and decentralized applications.

The issues of the cryptocurrencies legal status are a globaltask both within the framework of private and public law. According to Art. 128 of the Civil Code of the Russian Federation, the objects of civil rights include: things, including cash and documentary securities; other property, including property rights, including non-cash funds, uncertified securities, digital rights; results of work and services; protected intellectual activity results and means of individualization equated to them (intellectual property); intangible goods.

Due to the fact that cryptocurrency mainly performs the function of payment, it can be an equivalent, serving as a measure of the value for any goods and services that can be directly exchanged for them, then by its economic essence it refers to financial assets according to accounting standards [5]. Therefore, it will be in the asset of the organization's balance sheet and therefore belongs to the property. Therefore, following this logic, cryptocurrency can be classified according to Art. 128 of the Civil Code of the Russian Federation as other property.

The Ninth Arbitration Court of Appeal in case No. A40-124668/2017 indicated that according to Art. 18 of the Civil Code of the Russian Federation, citizens can have property by right of ownership, make any transactions that do not contradict the law and participate in obligations, as well as have other property rights. Due to the dispositiveness of the 
regulations of civil law in the Civil Code of the Russian Federation, there is no closed list of the civil rights objects under Art. 128 of the Civil Code of the Russian Federation. The current civil legislation does not contain the concept of "other property" mentioned in article 128 of the Civil Code of the Russian Federation, taking into account modern economic realities and the level of information technologies development, its broadest interpretation is permissible. According to the court, cryptocurrencies can be regarded as other property under Art. 128 of the Civil Code of the Russian Federation. Therefore, any property of the debtor that has economic value for the creditors (including cryptocurrency) cannot be arbitrarily excluded from the bankruptcy estate. Therefore, the court decided to transfer access to the crypto wallet (transfer the password) to the financial manager.

According to Russian legislation, transactions using cryptocurrency are not protected by Russian laws. However, one of the principal features that distinguishes crypto-money from such money is the way it appears in the digital space. So real means of payment must first be deposited into a specific account or electronic wallet, and cryptocurrency units appear already in electronic form. The issue of "digital money" comes literally from the Internet. Therefore, we can conclude that a cryptocurrency is a certain set of symbols of characters contained in an information system, while access to the information system is carried out using an information and telecommunication network using special software.

Since the concept of cryptocurrency is not defined by the current legislation, there are no special requirements for the procedure for its circulation, the legal status of cryptocurrencies is not defined, the essence of relations associated with the circulation of cryptocurrencies does not allow applying the norms regulating similar relations to cryptocurrencies. Since there is no concept of cryptocurrency in the legislation, it is impossible to unambiguously determine which category it belongs to: "property", "asset", "surrogate", "information" [9]. Thus, it does not seem to settle the relationship related to cryptocurrency. Consequently, the cryptocurrency can be regarded in relation to Art. 128 of the Civil Code of the Russian Federation otherwise than other property.

\section{Results and discussions}

Many provisions of real law will be difficult when applied to cryptocurrency, in particular, a difficult issue is an issue of determining the ownership of a thing. Also, a vindication claim will not be applicable for cryptocurrency due to the fact that there is no gap between the actual possession of a thing and its rights (here they are the same). A negative claim in relation to cryptocurrency is unlikely due to the fact that the blockchain platform is a peerto-peer system without any intermediaries. Therefore, to use cryptocurrency, you must have open-source software and a private key from the wallet. Consequently, there are no situations when there are obstacles related to the exercise of the powers to use and dispose the cryptocurrency.

Losing the ability to own a cryptocurrency means losing the ability to manage your crypto wallet as a result of losing your password. At the same time, it is impossible to physically lose cryptocurrency, since it does not exist in the material world, so it is doubtful to speak of cryptocurrency as a commodity, even as a digital one.

Cryptocurrency has the characteristics of many civil rights objects and at the same time does not belong to any of them and does not correspond to anyone in a complete way. This is due to the uniqueness of cryptocurrency as a phenomenon itself. The mining process is not goods production or funds emission, it is the process of adding new blocks to the blockchain. The meaning of the mining process is not in the "emission" and creation of the cryptocurrency as such, but in the verification of the blocks, for which the miners are rewarded. Therefore, the actions of the miner can be attributed to legal actions, that is, to the legitimate actions of the subject of law, which are not specifically aimed at the emergence, change or termination 
of legal relations, but entails such consequences. Therefore, it is not the technical process of mining itself that should be regulated, but its results - those cryptocurrencies received as a reward for the work.

Users of crypto-wallets can use the accumulated cryptocurrency as monetary assets (when paying with cryptocurrency for goods, works, services); financial investments (when investing using crypto tokens (ICO), derivatives converted into cryptocurrency, contributions to the authorized capital of organizations).

Cryptocurrency transactions are bearer transactions, that is, they operate in a similar way to cash or bearer securities, so when a transaction is completed it is irrevocable. All transactions are traced back to the public key. If the owner loses the private key, then he loses the right to the cryptocurrency, the contents of the wallet will no longer be available to anyone.

Cryptocurrency as an asset has such new and distinctive features as intangibility, cryptographic authentication, decentralization, management through consensus, the use of distributed ledgers. It was they who caused most of the disputes over the legal and property status of cryptocurrencies.

Taking into account the analysis of the cryptocurrencies essence in the US [6], Philippines [7] and Great Britain [8], the authors propose the following definition of a cryptocurrency.

Cryptocurrency is a cryptographically protected property that exists exclusively in digital form in an information system organized using distributed ledger technology, which can be stored, transferred and traded electronically.

The definition proposed above emphasizes the exclusively digital version of the existence and use of the object, indicates the circulation possibility for this asset, delimits the concept of cryptocurrency, non-cash and electronic money.

However, the very term cryptographically protected property describes it is necessary to describe not the thing itself as such, but also the legal relations that are associated with it. One of the first and most important issues is the ownership recognition for this specific type of property. Whether or not the right to recognize ownership of a cryptocurrency ultimately depends on the recognition of cryptocurrency as an object of civil rights and the developed regulatory norms of the law. According to Art. 209 of the Civil Code of the Russian Federation, the owner owns the rights of ownership, use and disposal of property. He may, at his discretion, perform actions in relation to the property belonging to him, including alienate the property to other persons, transfer the rights of ownership, use and disposal, while remaining the owner, pledge and burden in other ways.

It can already be noted that the cryptocurrency has signs of ownership. Such new and distinctive features of this asset as intangibility, cryptographic authentication, etc. do not deprive the cryptocurrency of this right. Crypto-assets cannot be deprived of being in the ownership regime just because they cannot be classified as intangible or tangible assets in accordance with current legislation. Determination of ownership for cryptocurrency can be very important in the future for the rules of law governing legal succession cases, such as inheritance, bankruptcy, fraud, theft, and breach of trust.

Due to the fact that cryptocurrency does not exist in physical material form, being only "virtual", from a legal point of view, it cannot be a subject of collateral. Also, the cryptocurrency does not have the title documents.

According to the authors, the starting point for determining the ownership of the cryptocurrency should be that the subject of the right will be the owner of this asset if he legally gained access to the private key by analogy as the subject acquired the ownership of a tangible asset on a legal basis.

The division of rights into property and liability has been the main classification of rights to things since the time of Roman law. Cryptocurrency rights do not belong to real rights, since this object is not tangible and therefore cannot be in physical possession. In this case, 
knowledge of the private key is not enough, which gives practical control over the wallet. Real law is associated with the physical control of material objects, and practical control includes a broader concept that extends both to intangible assets and to things that the law would not consider ownership at all.

Whether the rights to cryptocurrency will belong to the law of obligations is debatable. According to paragraphs 1, 2 of Art. 307 of the Civil Code of the Russian Federation, by virtue of an obligation, one person (the debtor) is obliged to perform a certain action in favor of another person (the creditor), such as: transfer property, perform work, provide a service, contribute to joint activities, pay money, etc., or to refrain from a certain action, and the creditor has the right to demand that the debtor fulfill his obligation. Obligations arise from contracts and other transactions, as a result of harm, as a result of unjust enrichment and other grounds.

A cryptocurrency will probably not fully fit this definition. In a completely decentralized system such as Bitcoin, the participants do not make any legal obligations towards each other. However, the law of obligations is a broader concept, that is, in fact, everything that does not belong to the real right.

The issue of restoring property rights in the case of a technically lost cryptocurrency (lack of access to the private key) is also unresolved. This problem has not been solved not only from the legal but also from the technical side of the issue. If the secret key can be recovered with the help of the third parties, then there is a vulnerability risk in terms of illegal seizure of the secret key. On the other hand, if we consider it by analogy with bearer securities or order securities, the Civil Procedure Code of the Russian Federation allows the rights restoration under such securities. The legislator dedicated chapter 34 of the Code of Civil Procedure to this - procedure to declare lost documents void. In case of loss of bearer securities, the court shall recognize them as invalid and at the same time restore the rights under them in the absence of a dispute about the right.

With its legal and economic properties, cryptocurrency is subject to illegal seizure. The most widespread seizure of this asset is seizure by means of fraud (identity theft, mass marketing scams, the use of imitators, phishing, the use of malware, personal extortion). By simplifying the user interface, the number of crypto assets users will increase, as well as the number of crimes associated with cryptocurrency.

\section{Conclusion}

1. The modern stage of social and economic society development is characterized by the digitalization processes and shows a significant advance of the scientific and technical rulemaking processes, which ensures the legal regulation of social relations.

2. It should be noted that there is a tendency for the courts to recognize the existence of such phenomena as mining, cryptocurrency, token, ICO in civil, arbitration and criminal proceedings. The definition of the essence of the cryptocurrency as an object of civil law will determine its status as an object and means for encroachments and committing socially dangerous acts.

3. When classifying a cryptocurrency as incorporeal, you can determine its ownership by legal obtaining a private key.

4. The criminal use of cryptocurrency can be explained both by the difficulty of its qualification and by the technological features of its existence exclusively in digital form. Cryptocurrency is used both as a mean of committing a crime and as a subject of a crime. With the simplification of the user interface and access to innovative blockchain technology, the number of such crimes related to cryptocurrency as theft, fraud, robbery, plunder, extortion, embezzlement, and commercial bribery will increase.

5. The economic and legal relations transformation affects the process of introducing new 
innovative digital technologies. The transition of socio-economic relations to a virtual network entails a change in regulation. Cryptocurrencies are a powerful tool for creating a decentralized peer-to-peer payment system. Unlike ordinary money, counterfeiting of cryptocurrencies is impossible and is determined by the interaction protocol between network participants. The result of using blockchain technology is an alternative to government regulation with its own set of rules and regulations.

\section{References}

1. I. Bashir, Blockchain: architecture, cryptocurrencies, development tools, smart contracts, 35 (2019)

2. L.V. Sannikova, Yu.S. Kharitonova, Law and Economics, 4 (2019)

3. Internet portal: "Blockchain.com", https://www.blockchain.com/

4. Internet portal: "CoinMarketCap", https://coinmarketcap.com/

5. I.A. Astrakhantseva, R.G. Astrakhantsev, A.S. Savina, Izvestia of higher educational institutions, Series "Economics, finance and production management", 4(42), 3 (2019)

6. New York Codes, Rules and Regulations, Title 23. Part 200 "Virtual Currencies", https://govt.westlaw.com/

7. Internet portal: "Coindesk", https://www.coindesk.com/

8. Legal statement on cryptoassets and smart contracts UK Jurisdiction Taskforce, https://www.lexology.com/

9. A.V. Savelyev, Law, 8, 136 (2017) 